\title{
Assessing hospital competition when prices don't matter to patients: the use of time-elasticities
}

\author{
Marco Varkevisser • Stéphanie A. van der Geest • \\ Frederik T. Schut
}

Received: 5 September 2008 / Accepted: 7 July 2009 / Published online: 7 August 2009

(C) The Author(s) 2009. This article is published with open access at Springerlink.com

\begin{abstract}
Health care reforms in several European countries provide health insurers with incentives and tools to become prudent purchasers of health care. The potential success of this strategy crucially depends on insurers' bargaining leverage vis-à-vis health care providers. An important determinant of insurers' bargaining power is the willingness of consumers to consider alternative providers. In this paper we examine to what extent consumers are willing to switch hospitals when they are fully covered for hospital services, which is typical for many European countries. Since prices do not matter to these patients, we estimate time-elasticities to assess hospital substitutability. Using data from a large Dutch health insurer on nonemergency neurosurgical outpatient hospital visits in 2003, we estimate a conditional logit model of patient hospital choice taking both patient heterogeneity and hospital characteristics into account. We use the parameter estimates to simulate the demand effect of an artificial increase in travel time by $10 \%$ for every patient, holding all other hospital attributes constant. Overall, the resulting point estimates of hospitals' time-elasticities are fairly high, although variation is substantial $(-2.6$ to -1.4$)$. Sensitivity tests reveal that these estimates are very robust and differ significantly across individual hospitals. This implies that all hospitals in our study sample have at least one close substitute which is an important precondition for effective hospital competition.
\end{abstract}

Keywords Hospital competition · Time elasticities · Patient choice $\cdot$ Health care reform

JEL Classification $\quad \mathrm{C} 25 \cdot \mathrm{I} 11 \cdot \mathrm{D} 12$

\footnotetext{
M. Varkevisser $(\varangle) \cdot$ S. A. van der Geest · F. T. Schut Institute of Health Policy \& Management (iBMG), Erasmus University Rotterdam, P.O. Box 1738, Room J8-03, 3000 DR Rotterdam, The Netherlands e-mail: varkevisser@bmg.eur.nl
} 


\section{Introduction}

After decades of central price and capacity control, several European governments are now reforming their health care system by introducing competition. In countries like Germany, the Netherlands, and Switzerland competition has recently been introduced in social health insurance to motivate health insurers to act as prudent purchasers of health care. Differential and selective contracting of providers is expected to encourage providers to reduce prices of services, increase quality and to better tailor services to consumer needs. In order to obtain these benefits it is important that health insurers have sufficient bargaining power vis-à-vis health care providers. Empirical evidence indicates that the willingness of consumers to switch providers is most important in determining insurers' abilities to negotiate favourable contracts (Sorensen 2003). As in any market, the intensity of competition among health care providers will therefore be driven by consumers' preferences for different providers.

To assess the feasibility of effective provider competition typically the price-elasticity of demand for individual providers is estimated. If the demand faced by an individual provider is price-inelastic, the provider has a strong market position which may hamper competition. But what if prices do not matter to consumers? In the Netherlands as well as in many other European countries health insurance coverage is very comprehensive and out-of-pocket payments are either absent or do not differ across provider alternatives. This implies that consumers are insensitive to differences in price. In addition, as opposed to the United States, consumers in Europe typically do not commit to a restricted provider network when buying health insurance. ${ }^{1}$ Under these circumstances, providers compete directly for patients by non-price factors only and travel time is then the "price" consumers face when selecting a health care provider (Varkevisser et al. 2008).

In this paper we argue that when monetary prices do not matter to consumers, provider substitutability that underlies each provider's individual bargaining clout can be assessed by estimating time-elasticities. A provider's time-elasticity measures consumers' propensity to switch to other providers in response to an artificial increase in travel time. A relatively low time-elasticity implies that compared to other providers only a few patients are likely to switch when that particular provider would become more costly (in terms of travel costs) and therefore less attractive, suggesting the absence of close substitutes. In that case, the provider has a stronger bargaining position than other providers which lowers the health insurer's ability to negotiate price discounts and/or quality improvements. It is more difficult for an insurer to divert patients from a provider with time-inelastic demand than to divert patients from a provider with time-elastic demand. Time-elasticities thus at least have an ordinal meaning. Assuming that patients are willing to trade time for money at a constant rate, price-elasticities are directly proportional to time-elasticities (Capps et al. 2001). However, since we do not know patients' time-money trade-off there is no cardinal meaning to the time-elasticities we estimate in this paper.

Computing time-elasticities requires the estimation of a patient choice model from which the demand for each provider can be derived. In this paper, we use a unique data set from a large Dutch health insurer on non-emergency neurosurgical outpatient hospital visits in 2003 to analyse patients' preferences for hospitals. We assume that patients visit the

\footnotetext{
1 Models specifically designed to estimate the value consumers place on different provider networks (Town and Vistnes 2001; Capps et al. 2003) do therefore not accurately depict markets for health care in European countries. Switzerland may be an exception, since there integrated insurer-provider organisations have a significant and increasing market share.
} 
hospital that maximizes their utility given their own characteristics and the characteristics of the hospitals (e.g. travel time to the hospital, quality) in their choice set. Based on this theoretical framework, we estimate a conditional logit model (McFadden 1974). A hospital's time-elasticity is then computed by simulating the demand effect of an artificial increase in travel time to the hospital by $10 \%$ for every patient, holding all other attributes constant. Our results show that patient demand for neurosurgery is rather time-elastic, but estimated time-elasticities differ significantly across individual hospitals. Hence, in the market for neurosurgery some hospitals face fewer close substitutes than others and therefore have a stronger market position. Information about hospital substitutability revealed by the estimation of time-elasticities is relevant for assessing the feasibility of selective contracting by health insurers and also for assessing proposed hospital mergers by antitrust enforcement agencies.

Our contribution to the existing literature is twofold. First, we provide a test for the feasibility of effective competition among health care providers in a setting where prices do not matter to consumers. Studies on patient hospital choice are almost exclusively performed in the context of US hospital markets, which is quite different from the more regulated health care setting in most European countries. We show how predicted patient flows following an artificial change in travel time can be used to identify which hospitals are competitors. Second, unlike previous studies that only present point estimates of hospitals' demand elasticities we use a parametric bootstrap method to obtain confidence intervals for the estimated time-elasticities.

The remainder of this paper is structured as follows. First, we describe the regulated context in which during our study period hospital prices were determined in the Netherlands after which a brief overview of the existing empirical literature on patient hospital choice is presented. Next, we describe our conditional logit model of patient hospital choice and the data used for the empirical analysis. Then we discuss the time-elasticities of Dutch hospitals based on our simulation results and the results of the robustness tests. Finally, we offer some concluding remarks.

\section{Price determination in the Dutch hospital sector}

From 1983 to 2005 per diem rates for Dutch hospitals were derived from a global budget (Schut and Van de Ven 2005). The hospital budget was partly fixed and partly determined by the volume of production that each hospital had to negotiate with health insurers. If actual hospital production exceeded the ex ante negotiated level of output, next year's prices had to be reduced to compensate for the resulting difference between a hospital's revenue and its budget. For each hospital, all health insurers paid the same price (per diem rate). During this period, people entitled to social health insurance (two thirds of the total population) were fully covered for hospital care. Hence, for the majority of the Dutch population hospital prices did not matter. As a result of price regulation, however, Dutch patients' price-insensitivity and the absence of selective contracting did not result in arbitrarily high prices for hospital services.

Only since 2005 hospitals and health insurers are able to freely negotiate prices, service, and quality for part of the hospital services. From 2005 to 2009 the proportion of the freely negotiable hospital production has been increased from 10 to $34 \%$ of total hospital expenditure. 


\section{Empirical literature on patient hospital choice: a brief overview}

Most of the early literature on patient hospital choice examines patterns of hospital utilisation using spatial interactions models, most commonly in the form of gravity models. Such models hypothesise that a greater level of spatial interaction between two areas is expected when the population masses of those areas are larger and the spatial distance between them is smaller. Representative studies include Morill et al. (1970), Roghmann and Zastowny (1979), and McGuirk and Porell (1984). Since the mid-eighties, however, researchers have used random utility theory to analyse in more detail why patients prefer a particular hospital to others (e.g. Folland 1983; Lee and Cohen 1985). The widespread availability of individual patient level utilisation data in the United States enabled the development of sophisticated econometric methods to estimate the probability that patients will be admitted to a particular hospital, explicitly incorporating the existence of other hospitals. ${ }^{2}$ From these studies it follows that, in addition to distance or travel time, both hospital and patient attributes have a substantial impact on hospital admission choices. In particular hospital quality seems to affect patient choice. It is important to notice that most studies reveal the trade-off between travel time and hospital quality to vary with patient characteristics. These studies include Bronstein and Morrisey (1991), Burns and Wholey (1992), Phibbs et al. (1993), Hodgkin (1996), Tay (2003), Tai et al. (2004), and Howard (2005). From these findings it can be concluded that hospitals offer a differentiated product to a segmented market. As a result, simply aggregating all patients may lead to biased estimation results, even when these patients suffer from the same specific medical condition.

\section{Conditional logit model of patient hospital choice}

The model

Our model is based on standard random utility theory. It uses a patient-level utility function in which travel time and hospital attributes reflecting quality differences are the main determinants of patient hospital choice. When selecting a hospital, patients are assumed to weigh the costs of increased travel time (including both monetary costs as well as the opportunity costs of time for themselves and/or their relatives) against the benefits (higher quality). The utility of patient $i$ who visits hospital $j$ is represented by

$$
U_{i j}=\delta t_{i j}+\sum_{k=1}^{n} \lambda_{k} H_{k j}+\sum_{l=1}^{m} \rho_{l} P_{l i} t_{i j}+\sum_{k=1}^{n} \sum_{l=1}^{m} \sigma_{k l} H_{k j} P_{l i}+\gamma_{i j}
$$

where $t_{i j}$ reflects travel time from patient $i$ 's home to hospital $j ; H_{. j}$ is a vector of hospital $j$ 's attributes; $P_{. i}$ is a vector of patient $i$ 's socio-economic characteristics; and $\gamma_{i j}$ represents the idiosyncratic part of patient $i$ 's evaluation of hospital $j$. Note that prices are not included in this function because we study patient choice in a setting where prices are irrelevant: patients are fully insured for hospital services without facing any out-of-pocket payments.

2 In their extensive review article, Porell and Adams (1995) summarise and assess the historical developments in hospital choice literature from the late 1960s until the early 1990s. 
We assert that patient $i$, given his needs and preferences, visits hospital $j$ when visiting any other alternative hospital would result in lower utility. ${ }^{3}$

By interacting travel time and hospital attributes with the individual patient characteristics, we allow the trade-off between travel time and hospital quality to vary across patients. ${ }^{4}$ When a particular hospital would become less attractive, its patients possibly make different decisions. Depending on their place of residence and other individual characteristics some patients may still decide to visit this hospital, while others may change to another nearby hospital or prefer to travel further for their hospital care. This flexibility is a major advantage of the model, because it allows us to analyse the substitutability of hospitals more precisely.

\section{Empirical specification}

For the empirical specification of Eq. 1, we define the dependent variable $H C_{i j}$ which is assigned the value 1 when patient $i$ visited hospital $j$ and the value 0 otherwise. For each patient a choice set of feasible hospital alternatives is defined $\left(N_{i}\right)$. We estimate Eq. 1 as a conditional logit model (McFadden 1974). It is therefore assumed that, for each patient, the relative probabilities of visiting any two hospitals are independent of any other available alternatives. This restriction, called the independence of irrelevant alternatives (IIA) assumption, implies that one assumes all systematic variation in patients' taste to be sufficiently captured by the explanatory variables incorporated into the logit model. The remaining, unobserved portion of utility $\varepsilon_{i j}$ is then essentially white noise. To test whether the IIA-assumption is valid in this context, we perform the Hausman-McFadden (1984) test. If this assumption is not violated patient $i$ 's probability of visiting hospital $j$ is represented by

$$
\operatorname{Pr}_{i j}=\frac{\exp \left(\delta t_{i j}+\sum_{k=1}^{n} \lambda_{k} H_{k j}+\sum_{l=1}^{m} \rho_{l} P_{l i} t_{i j}+\sum_{k=1}^{n} \sum_{l=1}^{m} \sigma_{k l} H_{k j} P_{l i}\right)}{\sum_{j=1}^{N_{i}} \exp \left(\delta t_{i j}+\sum_{k=1}^{n} \lambda_{k} H_{k j}+\sum_{l=1}^{m} \rho_{l} P_{l i} t_{i j}+\sum_{k=1}^{n} \sum_{l=1}^{m} \sigma_{k l} H_{k j} P_{l i}\right)}
$$

This study analyses choices concerning patients' first outpatient non-emergency hospital visits for neurosurgery. ${ }^{5}$ Since these visits to the hospital's outpatient clinic are patients' first appointment with a physician for initial consultation or examination, the severity of their illness is at that moment not yet fully known. ${ }^{6}$ The decision to visit hospital $j$ is expected to be negatively affected by the travel time from patient $i$ 's home. Based on the findings from previous research, we include four additional hospital attributes to test for their ability to attract patients: university medical centre, overall reputation, reputation for neurosurgery, and waiting time. Previous research revealed that Dutch patients are less likely to choose academic hospitals for their first hospital visit, whereas a shorter waiting time increases the attractiveness of a hospital (Varkevisser and Van der Geest 2007). Since in the Netherlands hospital clinical quality was not yet systematically measured in 2003 , in this paper indicators of hospitals' overall reputation and reputation for neurosurgery are included as a proxy for hospital quality as perceived by the patient and his advising GP.

3 In the Netherlands the decision which hospital to visit is made by patients themselves, most often in consultation with their general practitioner (GP). Since Dutch GPs do not face any economic incentives to refer patients to particular hospitals, it is not in their interest to ignore patients' preferences.

4 In this paper quality as perceived by the patient and his advising GP determines hospital choice, rather than hospitals' unobservable intrinsic clinical quality.

5 For emergency visits it is likely that patients simply choose the nearest hospital rather than the hospital that, given its attributes, would maximise their utility.

6 Neurosurgical conditions include primarily brain, spinal cord, vertebral column and peripheral nerve disorders. By far the most common procedure is surgery to correct a hernia. 
An issue that arises when estimating a model of patient hospital choice is the potential endogeneity of quality: does high quality attract patients or does a high number of patients lead to high quality? If the latter is true, hospitals with geographically attractive locations may gradually become high-quality hospitals over time. In our sample, however, there is no correlation between a hospital's reputation and its size. Hence, higher-quality hospitals are not systematically higher-volume hospitals. We therefore treat quality as exogenous in the model, so that the causation runs from quality to patient choice and not in reverse.

Another potential endogeneity problem may occur if hospital waiting time is affected by patients' hospital choice. This would be the case if consumers have a preference for hospitals with high waiting times because they interpret higher waiting times as a signal of higher quality. However, empirical evidence for the Netherlands (NZa 2007) as well as for other countries (e.g. Hanson et al. 2004; Martin et al. 2007) shows that patients tend to avoid hospitals with relatively high waiting times. We also do not observe any correlation between waiting time, overall reputation and reputation for neurosurgery in our data. Hence, high quality is here not associated with high waiting time. Therefore we treat each hospital's waiting time as an exogenous explanatory variable and expect a negative sign for it. ${ }^{7}$

All other things equal, patients are more likely to end up in large hospitals than in smaller ones. To control for this exogenous size effect, hospital $j$ 's total number of beds and its annual volume of first outpatient hospital visits for neurosurgery are also included as explanatory variables.

The patient characteristics in our model include gender, age (adult vs. non-adult), employment, and social status. ${ }^{8}$ The effect of gender on patients' hospital choice is unclear beforehand. Non-adult patients may be associated with a higher propensity to travel, since parents are likely to be less averse to travel when seeking the best neurosurgical hospital care available for their children.

Since all patients in our dataset are enrolled in social health insurance, their annual income during our study period (2003) did not exceed the threshold for eligibility (€31,750). Most patients are salaried employees (or their dependents). Those who are not, are identified by the following dummy variables: unemployment, retirement, incapacity for work, social security, and self-employment. Retired patients may be more averse to travel than others because they are more likely to have physical problems restricting their mobility. More distant hospitals are also expected to be less attractive to unemployed patients and patients on social security. Given their low income, it is likely that they are less willing to incur travel expenses. Patients who are incapacitated for work are likely to have a serious chronic condition which could make them more sensitive to (perceived) hospital quality differences. Hence, they may be more willing to travel to a distant but better performing hospital. On the other hand, these patients are also likely to have physical impairments that may reduce their propensity to travel. Finally, self-employed patients may be less willing to travel because they are likely to have higher opportunity costs of (travel) time since - in contrast to salaried employees - their income is directly related to actual working time. ${ }^{9}$

\footnotetext{
7 Nevertheless, if hospital waiting time - contrary to our expectations-would be perceived as a high quality signal, patients' disutility of waiting time is likely to be underestimated. We do not have sufficient data to construct reliable instrumental variables to correct for this potential bias. As long as we find hospital waiting time to be negatively correlated with hospital choice, this potential bias may not be a serious problem.

8 Patients' own previous experiences with hospitals may also affect their decisions. Unfortunately, such information is lacking in our dataset.

9 As a result of collective labour agreements, nearly all salaried employees in the Netherlands have the right to visit a doctor during working time without loss of income.
} 
Data

Our principal data source is the Agis Health Database that contains individual patient level data from a large Dutch health insurer (Agis). In 2003, Agis was one of the largest Dutch health insurers representing approximately 1.4 million socially insured amounting to a nationwide market share of $14 \%$. Its key geographical areas include both urban (Amsterdam, Utrecht) and rural areas. Samples from this database are representative for the Dutch socially insured population (two thirds of the total population). Since all patients in the sample are enrolled in a social health insurance plan no-one has to pay an out-of-pocket price for hospital services.

From the Agis Health Database we obtained observations on non-emergency first outpatient hospital visits for neurosurgery in 2003. In addition to each patient's socio-economic characteristics, the available data include the zip code and name of the hospital visited. There are 66 hospitals in the Netherlands providing neurosurgery. The most common types of neurosurgery (such as hernia repairs) are performed in all these hospitals.

Patients' travel times are defined as the fastest route by car from patient $i$ 's home to each hospital and are calculated in minutes. This route is obtained from a database that includes all 4-digit zip codes in the Netherlands and accounts for differences in average speed that exist between different road types. ${ }^{10}$ If patients travelled extremely long to the hospital visited, it can reasonably be assumed that they were away from home when they needed health care. For those patients, travel time from their place of residence incorrectly measures the travel costs incurred, which may bias the estimation results. In this paper we therefore exclude all patients who travelled more than $60 \mathrm{~min}(4.6 \%)$. For the remaining patients a set of hospital choices is defined $\left(N_{i}\right)$. Each patient's choice set consists of all hospitals providing neurosurgery that can be reached within $60 \mathrm{~min}$ of travel time. Note that on average patients travelled 19 min, while for $95 \%$ of the patients actual travel time did not exceed $45 \mathrm{~min}$. More than $80 \%$ travelled less than $30 \mathrm{~min}$. In the Netherlands, even patients who need a very complex neurosurgical treatment do not have to travel more than $60 \mathrm{~min}$ to the nearest hospital capable of performing the surgery. Hence, within the Dutch context a limit to each patient's hospital choice set of 60 min of travel time is not likely to be restrictive when analysing actual choice behaviour. Since Dutch health insurers were not allowed to contract selectively with hospitals in 2003 there are no further restrictions on patients' choice sets.

The resulting study sample contains 5,389 individual patient hospital visits. From Table 1 it follows that for almost $90 \%$ of these patients the choice set includes at least 15 hospitals. Only little more than $2 \%$ of the patients can reach at most five hospitals within 60 min of travel time from their home. On average, patient $i$ 's choice set includes 26 hospitals. Average travel time to the nearest hospital providing neurosurgery is $13 \mathrm{~min}$. Varkevisser and Van der Geest (2007) found that for their first outpatient hospital visit 54\% of the neurosurgery patients bypassed the nearest hospital alternative. For these patients bypassing the nearest hospital resulted in about $11 \mathrm{~min}$ more travel time than would be strictly necessary.

As already mentioned, a lack of data forces us to use indicators such as hospitals' reputation as a proxy for quality. However, we do not consider this to be a serious shortcoming. Due to the absence of clinical quality indicators during the study period, patients at that time also had to rely on (information about) hospitals' reputation for making choices. Information on hospital's overall reputation and reputation for neurosurgery are both taken from an annual survey among Dutch physicians, nurses and hospital managers published in a popular news magazine (Elsevier 2003). The explanatory variable reflecting each hospital's overall

10 As Dutch zip codes consist of four numbers followed by two letters (e.g. $3000 \mathrm{DR}$ ), the maximum inaccuracy in distance between this point and the actual starting point and destination could be about $250 \mathrm{~m}$ in urban areas and 1,000 $\mathrm{m}$ in rural areas at most. 
Table 1 Number of hospitals in patients' choice sets

\begin{tabular}{|c|c|c|c|}
\hline Hospitals (\#) & Patients (\#) & Patients (\%) & Patients (cum. \%) \\
\hline 1 & 1 & 0 & 0 \\
\hline 2 & 20 & 0 & 0 \\
\hline 3 & 31 & 1 & 1 \\
\hline 4 & 30 & 1 & 2 \\
\hline 5 & 40 & 1 & 2 \\
\hline 6 & 74 & 1 & 4 \\
\hline 7 & 68 & 1 & 5 \\
\hline 8 & 43 & 1 & 6 \\
\hline 9 & 90 & 2 & 7 \\
\hline 10 & 39 & 1 & 8 \\
\hline 11 & 19 & 0 & 8 \\
\hline 12 & 28 & 1 & 9 \\
\hline 13 & 26 & 1 & 9 \\
\hline 14 & 78 & 1 & 11 \\
\hline 15 & 9 & 0 & 11 \\
\hline 16 & 26 & 1 & 12 \\
\hline 17 & 88 & 2 & 13 \\
\hline 18 & 74 & 1 & 15 \\
\hline 19 & 33 & 1 & 15 \\
\hline 20 & 198 & 4 & 19 \\
\hline 21 & 176 & 3 & 22 \\
\hline 22 & 256 & 5 & 27 \\
\hline 23 & 112 & 2 & 29 \\
\hline 24 & 165 & 3 & 32 \\
\hline 25 & 219 & 4 & 36 \\
\hline 26 & 88 & 2 & 38 \\
\hline 27 & 97 & 2 & 40 \\
\hline 28 & 394 & 7 & 47 \\
\hline 29 & 541 & 10 & 57 \\
\hline 30 & 779 & 15 & 71 \\
\hline 31 & 155 & 3 & 74 \\
\hline 32 & 364 & 7 & 81 \\
\hline 33 & 258 & 5 & 86 \\
\hline 34 & 149 & 3 & 89 \\
\hline 35 & 210 & 4 & 92 \\
\hline 36 & 158 & 3 & 95 \\
\hline 37 & 46 & 1 & 96 \\
\hline 38 & 79 & 2 & 98 \\
\hline 39 & 60 & 1 & 99 \\
\hline
\end{tabular}


Table 1 continued

Each patient's choice set comprises all hospitals providing neurosurgery within $60 \mathrm{~min}$ of travel time

\begin{tabular}{llll}
\hline Hospitals (\#) & Patients (\#) & Patients (\%) & Patients (cum. \%) \\
\hline 40 & 61 & 1 & 100 \\
41 & 7 & 0 & 100 \\
Sum & 5,389 & 100 & \\
Mean & 26 & & \\
Modus & 30 & & \\
\hline
\end{tabular}

reputation measures several hospital-specific items, including both positive items (e.g. attitude towards patients, medical and nursing staff expertise, facilities) and negative items (e.g. financial problems, medical staff disputes, high staff turnover). This dummy variable has the value 1 if hospital $j$ 's unweighted sum of its bonus and penalty points exceeds the national average and the value 0 otherwise. The reputation for neurosurgery is also captured by a dummy variable. This variable has the value 1 if hospital $j$ is recommended by at least $33 \%$ of the respondents and the value 0 otherwise.

Data on individual hospital waiting times is obtained from the Netherlands Hospital Association. In 2003, this data was accessible for patients by the internet and also frequently published in a Dutch newspaper. To test our hypothesis that hospitals with lower waiting times are more attractive to patients than other hospitals, we use a dummy variable that has value 1 when hospital $j$ 's waiting time for a neurosurgical first hospital visit was reported to be below the national average in 2003 and the value 0 otherwise.

Finally, we control for exogenous hospital size effects by including two additional explanatory variables on hospital size that are obtained from the Dutch Ministry of Health, Welfare and Sports. To capture possible diagnosis-specific size effects, we did not only include hospital $j$ 's total number of beds but also its annual number of first outpatient hospital visits for neurosurgery. This variable includes patients from all social health insurance plans and private insurers.

Table 2 reports the descriptive statistics of all variables that are included in our logit specification.

\section{Estimation results}

For the conditional logit estimation of Eq. 1 we use the maximum likelihood estimation method and standard iterative procedures for its optimisation. Maximum likelihood estimation of the conditional logit model can be shown under very general conditions to provide estimators that are asymptotically efficient and normally distributed (McFadden 1974). In particular when the dataset has many combinations of hospitals and residency zip codes with no actual visits ("zero flows"), maximum likelihood is the preferred estimation method for conditional logit models of patient hospital choice. As shown by Garnick et al. (1989), maximum likelihood estimates are far less sensitive to the presence of zero flows than alternative estimation techniques.

As already mentioned, we perform the Hausman-McFadden test to test the validity of the IIA assumption. This chi-squared test compares the estimated parameters and covariance matrices from the full choice set with a restricted choice set. For this study, the test statistic indicates we cannot reject the null hypothesis that the difference in coefficients is not systematic. Hence, the IIA assumption seems to hold here implying that all systematic variation in patients' taste is sufficiently captured by the explanatory variables. 
Table 2 Descriptive statistics of the study sample

\begin{tabular}{|c|c|c|c|c|}
\hline Variable & Mean & SD & Minimum & Maximum \\
\hline Actual travel time (in minutes by car) & 19 & 12 & 0 & 60 \\
\hline \multicolumn{5}{|l|}{ Patient attributes $(n=5,389)$} \\
\hline Female & 0.59 & & 0 & 1 \\
\hline Non-adult & 0.04 & & 0 & 1 \\
\hline Retired & 0.25 & & 0 & 1 \\
\hline Unemployed & 0.02 & & 0 & 1 \\
\hline Incapacitated for work & 0.25 & & 0 & 1 \\
\hline Social security & 0.06 & & 0 & 1 \\
\hline Self-employed & 0.02 & & 0 & 1 \\
\hline \multicolumn{5}{|l|}{ Hospital attributes $(n=66)$} \\
\hline University medical centre & 0.12 & & 0 & 1 \\
\hline Good overall reputation & 0.48 & & 0 & 1 \\
\hline Good reputation for neurosurgery & 0.09 & & 0 & 1 \\
\hline Waiting time below average & 0.39 & & 0 & 1 \\
\hline Total hospital beds & 611 & 268 & 210 & 1,368 \\
\hline First hospital visits for neurosurgery & 655 & 591 & 0 & 2,380 \\
\hline
\end{tabular}

In order to measure goodness of fit we analyse predicted versus actual hospital choice. Following Town and Vistnes (2001), a "hit-or-miss" criterion is constructed where the predicted choice for a patient is the hospital having the maximum predicted probability. The model correctly predicts $43 \%$ of patients' hospital choices. Given the large number of hospital alternatives available to patients (on average 26), this prediction rate suggests the model provides a high degree of explanatory power. The hospital choice model estimated by Town and Vistnes (2001) correctly predicts about 30\% of hospital choices for their different samples. Most studies on patient hospital choice, however, do not report how well their estimated models predict patients' actual choices. By exception, Capps et al. (2001) report that $35 \%$ of the patients in their sample visited the hospital having the maximum predicted probability.

Detailed estimation results are presented in Table 3. ${ }^{11}$ The coefficient for travel time is negative and statistically significant at $1 \%$ indicating that in general patients are averse to travel.

The estimation results for the hospital attributes show that patients are generally less likely to choose a university medical centre for their first outpatient neurosurgical hospital visit. There are two plausible explanations for this. First, for non-tertiary care Dutch physicians, nurses and hospital managers do not classify university medical centres among the best hospitals they know (Elsevier 2003) which may explain patients' preferences for general hospitals. Second, patients may expect to get more personal attention in a general hospital than in a university medical centre that is aimed at scientific research as well as medical education and where it is more likely for them to be (initially) treated by a medical resident

11 We also estimated a full model including all possible interactions of patient characteristics and hospital attributes. The likelihood-ratio test indicates that the full model is statistically preferred over the reduced one. However, we prefer to use the latter since most interaction variables included in the full model are insignificant and the reduced model's estimated parameters are more easy to interpret, while both the overall model performance and predicted market shares do not substantially differ between both models. 
Table 3 Estimation results conditional logit model

\begin{tabular}{|c|c|c|c|}
\hline ble 3 Estimation results & & Coefficient & SE \\
\hline & \multicolumn{3}{|l|}{ Travel time } \\
\hline & Travel time & $-0.1600 * *$ & 0.0037 \\
\hline & \multicolumn{3}{|l|}{ Hospital attributes } \\
\hline & University medical centre & $-1.7820^{* *}$ & 0.0870 \\
\hline & Good overall reputation & $0.1101 *$ & 0.0448 \\
\hline & Good reputation for neurosurgery & 0.0161 & 0.0599 \\
\hline & Waiting time below average & $0.3397 * *$ & 0.0356 \\
\hline & Total hospital beds & $0.0016^{* *}$ & 0.0001 \\
\hline & First hospital visits for neurosurgery & $0.0018 * *$ & 0.0001 \\
\hline & \multicolumn{3}{|l|}{ Interacted with Female } \\
\hline & Travel time & $-0.0105^{* *}$ & 0.0038 \\
\hline & University medical centre & -0.0164 & 0.0712 \\
\hline & \multicolumn{3}{|l|}{ Interacted with Non-adult } \\
\hline & Travel time & $0.0532 * *$ & 0.0085 \\
\hline & University medical centre & $4.1262 * *$ & 0.2804 \\
\hline & \multicolumn{3}{|l|}{ Interacted with Retired } \\
\hline & Travel time & $-0.0180 * *$ & 0.0052 \\
\hline & University medical centre & 0.1213 & 0.0916 \\
\hline & \multicolumn{3}{|l|}{ Interacted with Unemployed } \\
\hline & Travel time & 0.0135 & 0.0125 \\
\hline & University medical centre & -0.1924 & 0.2746 \\
\hline & \multicolumn{3}{|l|}{ Interacted with Incapacitated for work } \\
\hline & Travel time & $0.0171^{* *}$ & 0.0045 \\
\hline & University medical centre & $0.6456^{* *}$ & 0.0867 \\
\hline & \multicolumn{3}{|l|}{ Interacted with Social security } \\
\hline & Travel time & $-0.0257 * *$ & 0.0096 \\
\hline & University medical centre & 0.1249 & 0.1561 \\
\hline & \multicolumn{3}{|l|}{ Interacted with Self employed } \\
\hline & Travel time & 0.0174 & 0.0115 \\
\hline & University medical centre & 0.0118 & 0.2618 \\
\hline & Log likelihood & -8951.69 & \\
\hline & Degrees of freedom & 21 & \\
\hline & Number of patients & 5,388 & \\
\hline$* *$ Significance at $1 \% ; * 5 \%$ & Number of patient-hospital combinations & 142,037 & \\
\hline
\end{tabular}

$* *$ Significance at $1 \% ; * 5 \%$

instead of a fully qualified physician. From the estimation results it follows that a hospital's overall reputation significantly affects patients' choices. A good overall reputation increases a hospital's attractiveness. The estimated parameter for a hospital's reputation for neurosurgery is also positive, though not statistically significant. This may be due to the fact that only 6 of the 66 Dutch hospitals providing neurosurgery have a specifically good reputation for this medical speciality. Patients are in general also more likely to choose hospitals for which waiting time is below the national average.

From Table 3 it follows that several patient attributes significantly affect hospital choice, particularly in relation to travel time. Both female patients, retired patients, and patients on 
social security are significantly less inclined to travel. The opposite holds for non-adults and patients incapacitated for work. The latter are likely to suffer from chronic diseases and to have previous experiences with hospitals. This may explain why they are less averse to travel and more likely to visit a university medical centre. Our finding that also children are more likely to visit a university medical centre can be explained by the fact that in the Netherlands specialised facilities for paediatric care are concentrated in these hospitals. Overall, 26\% of the adult patients included in our study sample visit a university medical centre for their first neurosurgical outpatient visit, while this percentage for non-adult patients is $89 \%$.

\section{Using time-elasticities to assess hospital competition}

\section{Computing time-elasticities}

In this section we demonstrate how to compute time-elasticities for individual hospitals and use these to assess hospital substitutability. The underlying general methodology is obtained from Capps et al. (2001). Estimating the time-elasticity of hospital $j$ involves four successive steps. In the first step, hospital $j$ 's market share is predicted by summing up patients' estimated choice probabilities for hospital $j$. In the second step, travel time to hospital $j$ is artificially increased by a certain percentage (e.g. 10\%) for every patient while holding travel time to all other hospitals at their true level. This implies that patients are virtually moved away from hospital $j$ and brought relatively closer to other hospital alternatives in their choice set. These hospitals therefore become relatively more attractive to them. In the third step, hospital $j$ 's "new" market share is calculated. Using the estimated parameters from the patient choice model, new choice probabilities are calculated and summed up for hospital $j$ as well as all other hospitals to reveal substitution patterns. In the final step, hospital $j$ 's time-elasticity is computed dividing its percent change in predicted market share (number of patients) by the percent change in travel time. This exercise identifies the extent to which other hospitals are substitutes for hospital $j$, holding all other things equal. ${ }^{12}$

Since many of the 66 hospitals included in our dataset have individual market shares that are negligible, we only estimate time-elasticities for the hospitals with a predicted market share of at least $2.5 \%$ since those are located in Agis' key geographical areas. Together these hospitals have a predicted cumulative market share of almost $85 \%(n=13)$. Using the parameter estimates from Table 3, for each of these hospitals we predict the percent change in market share when travel time to that particular hospital is artificially increased by $10 \%$. From Table 4 it follows that the resulting time-elasticities show considerable differences. ${ }^{13}$

The highest time-elasticity (-2.6) is found for hospital 22. This general hospital looses 41 of its 158 predicted neurosurgical patients to other hospitals when travel time is artificially raised by $10 \%$ for every patient in our study sample. As revealed by the thirteenth column of Table 5, 10 patients switch to hospital 32 (24\%). The closest substitute for hospital 22, however, appears to be hospital 18 that is not included in the table but gains 13 patients (32\%) when patients" "price" for visiting hospital 22 increases by $10 \%$. Its relatively high time-elasticity

\footnotetext{
12 Buchmueller (2006) applies a quite similar approach when analysing the health plan choices of retirees. He uses the coefficient estimates from a conditional logit model for calculating the predicted change in each plan's markets share caused by a hypothetical $\$ 5$ increase in premium while holding the premiums of all competing plans constant. Since the observed prices differ considerably among the plans included in his analysis, the hypothetical percentage increase in premiums is (much) higher for the less expensive health plans. As a result, the predicted changes in market shares are ambiguous.

13 For reasons of confidentiality, hospital names are not revealed.
} 
Table 4 Estimated

time-elasticities

\begin{tabular}{llll}
\hline Hospital $j$ & $\begin{array}{l}\text { Predicted } \\
\text { patients (\#) }\end{array}$ & Travel time $+10 \%$ & \\
\cline { 3 - 4 } & & $\Delta$ Patients & Time-elasticity \\
\hline Hospital 56 & 714 & -137 & -1.9 \\
Hospital 32 & 669 & -99 & -1.5 \\
Hospital 52 & 550 & -85 & -1.5 \\
Hospital 46 & 464 & -91 & -2.0 \\
Hospital 37 & 344 & -63 & -1.8 \\
Hospital 03 & 315 & -69 & -2.2 \\
Hospital 59 & 293 & -63 & -2.1 \\
Hospital 63 & 248 & -53 & -2.1 \\
Hospital 14 & 242 & -52 & -2.2 \\
Hospital 39 & 239 & -33 & -1.4 \\
Hospital 22 & 158 & -41 & -2.6 \\
Hospital 48 & 149 & -31 & -2.1 \\
Hospital 64 & 136 & -30 & -2.2 \\
\hline
\end{tabular}

Table 5 Where do patients go if travel time to their first-best hospital is artificially increased?

\begin{tabular}{|c|c|c|c|c|c|c|c|c|c|c|c|c|c|c|}
\hline \multirow[t]{2}{*}{ Hospital $j$} & \multirow[t]{2}{*}{$\begin{array}{l}\text { Predicted } \\
\text { patients (\#) }\end{array}$} & \multicolumn{13}{|c|}{$\begin{array}{l}\Delta \text { Patients (\#) for each hospital when travel time to hospital } j \text { is increased } \\
\text { by } 10 \%\end{array}$} \\
\hline & & 56 & 32 & 52 & 46 & 37 & 03 & 59 & 63 & 14 & 39 & 22 & 48 & 64 \\
\hline Hospital 56 & 714 & -137 & 26 & 4 & 4 & 19 & 7 & 5 & 8 & 15 & 2 & 2 & 8 & 6 \\
\hline Hospital 32 & 669 & 32 & -99 & 4 & 5 & 8 & 5 & 3 & 14 & 9 & 2 & 10 & 3 & 7 \\
\hline Hospital 52 & 550 & 4 & 4 & -85 & 26 & 2 & 12 & 14 & 4 & 1 & 8 & & 1 & 2 \\
\hline Hospital 46 & 464 & 4 & 4 & 22 & -91 & 2 & 11 & 12 & 5 & 1 & 6 & & 1 & 2 \\
\hline Hospital 37 & 344 & 22 & 8 & 2 & 3 & -63 & 3 & 2 & 2 & 9 & 1 & & 7 & 2 \\
\hline Hospital 03 & 315 & 8 & 5 & 11 & 12 & 3 & -69 & 10 & 5 & 2 & 4 & & 1 & 2 \\
\hline Hospital 59 & 293 & 5 & 3 & 11 & 11 & 2 & 9 & -63 & 3 & 1 & 4 & & 1 & 1 \\
\hline Hospital 63 & 248 & 11 & 14 & 5 & 6 & 3 & 6 & 3 & -53 & 3 & 2 & & 1 & 4 \\
\hline Hospital 14 & 242 & 16 & 8 & 1 & 2 & 8 & 2 & 1 & 3 & -52 & 1 & & 4 & 2 \\
\hline Hospital 39 & 239 & 2 & 2 & 11 & 11 & 1 & 6 & 6 & 2 & 1 & -33 & & & 1 \\
\hline Hospital 22 & 158 & 2 & 7 & & & & & & & & & -41 & & \\
\hline Hospital 48 & 149 & 10 & 3 & 1 & 1 & 7 & 1 & 1 & 1 & 4 & & & -31 & 1 \\
\hline Hospital 64 & 136 & 8 & 8 & 2 & 2 & 2 & 2 & 1 & 5 & 2 & 1 & & 1 & -30 \\
\hline Other hospitals & 868 & 12 & 8 & 10 & 10 & 6 & 5 & 6 & 2 & 3 & 2 & 27 & 4 & 1 \\
\hline Sum & 5,388 & 0 & 0 & 0 & 0 & 0 & 0 & 0 & 0 & 0 & 0 & 0 & 0 & 0 \\
\hline
\end{tabular}

Since "fractional" patients are here rounded up/down to the nearest value, the columns are subject to round-off errors

implies that from all thirteen hospitals for which we have estimated time-elasticities hospital 22 potentially has the least bargaining power vis-à-vis health insurers.

For the hospital with the highest predicted market share, hospital 56, a time-elasticity with the value -1.9 is estimated. When we artificially increase travel time to this university 
Table 6 Assessing hospital substitutability (travel time $+10 \%$ )

\begin{tabular}{|c|c|c|c|c|c|c|}
\hline & \multirow[t]{2}{*}{$\Delta$ Patients } & \multirow{2}{*}{$\begin{array}{l}\text { Hospital } \\
\text { substitutes }^{a}\end{array}$} & \multicolumn{3}{|c|}{ Percentage patients switching to hospital $j$ 's } & \multirow[t]{2}{*}{$\mathrm{HHI}^{b}$} \\
\hline & & & $\begin{array}{l}\text { Closest } \\
\text { substitute (\%) }\end{array}$ & $\begin{array}{l}\text { Two closest } \\
\text { substitutes }(\%)\end{array}$ & $\begin{array}{l}\text { Three closest } \\
\text { substitutes (\%) }\end{array}$ & \\
\hline Hospital 56 & -137 & 7 & 24 & 40 & 52 & 0.119 \\
\hline Hospital 32 & -99 & 6 & 26 & 41 & 49 & 0.121 \\
\hline Hospital 52 & -85 & 5 & 26 & 39 & 53 & 0.131 \\
\hline Hospital 46 & -91 & 6 & 28 & 41 & 54 & 0.137 \\
\hline Hospital 37 & -63 & 4 & 30 & 43 & 55 & 0.143 \\
\hline Hospital 03 & -69 & 7 & 17 & 33 & 46 & 0.108 \\
\hline Hospital 59 & -63 & 6 & 22 & 40 & 56 & 0.130 \\
\hline Hospital 63 & -53 & 7 & 25 & 41 & 51 & 0.128 \\
\hline Hospital 14 & -52 & 5 & 28 & 46 & 63 & 0.154 \\
\hline Hospital 39 & -33 & 6 & 24 & 43 & 56 & 0.137 \\
\hline Hospital 22 & -41 & 4 & 31 & 56 & 67 & 0.179 \\
\hline Hospital 48 & -31 & 4 & 27 & 48 & 59 & 0.147 \\
\hline Hospital 64 & -30 & 7 & 24 & 43 & 57 & 0.135 \\
\hline
\end{tabular}

a Those hospitals that at least $5 \%$ of the patients substitute toward

$\mathrm{b}$ This HHI is a concentration measure for the diverted patients

medical centre by $10 \%$ for every patient, it looses 137 of its initially predicted 714 patients for neurosurgery. The closest substitutes for this hospital appear to be the hospitals 32, 37, and 14 . The number of patients substituting toward these alternative hospitals is 32,22 , and 16 , respectively. Jointly these three general hospitals located nearby adopt more than $50 \%$ of the patients leaving hospital 56 following an increase in "price" there.

The hospital with the lowest time-elasticity $(-1.4)$ is hospital 39: a medium-sized general hospital located in the inner city of Amsterdam. Only 33 of its predicted 239 patients switch to another hospital for their first neurosurgical outpatient visit when more travel time would be required. Patients apparently have relatively strong preferences for this hospital. Hence, in our study sample hospital 39 potentially has the best bargaining position with health insurers.

As revealed by Table 6, all hospitals in our study sample face substitutes. When a hospital would become less attractive, other hospitals will experience an increase in market share. If we identify competitors as those hospitals that at least $5 \%$ of the patients would substitute toward, Table 6 reveals that for all hospitals the number of competitors is fairly high. The minimum number of substitutes is 4 (hospitals 22, 37, and 48) and the maximum number is 7 (hospitals 3, 56, 63, and 64). However, the hospital substitution patterns found here reveal that overall about $25 \%$ of all switching patients are adopted by hospital $j$ 's closest substitute. The three closest substitutes jointly adopt more than half of the patients predicted to leave hospital $j$ following an artificial increase in travel time. Hence, the vast majority of hospital alternatives are only appealing to relatively few patients each. We therefore computed an HHI for the diverted patients. ${ }^{14}$ The lower the value of this HHI, the more scattered the patients substituting away from hospital $j$ are among the other hospitals. Note that if this

14 We thank one of the reviewers for making this suggestion. 
Table 7 Sensitivity tests estimated time-elasticities

\begin{tabular}{|c|c|c|c|c|c|c|}
\hline & \multicolumn{3}{|c|}{ Travel time $+10 \%$} & \multicolumn{3}{|c|}{ Travel time $+5 \%$} \\
\hline & \multicolumn{3}{|c|}{ Results parametric bootstrap $(n=1,000)$} & \multicolumn{3}{|c|}{ Results parametric bootstrap $(n=1,000)$} \\
\hline & Mean & $\mathrm{SD}$ & $95 \%$ Confidence & Mean & SD & $95 \%$ Confidence \\
\hline Hospital 22 & -2.6 & 0.02 & $-2.6,-2.6$ & -2.8 & 0.02 & $-2.9,-2.8$ \\
\hline Hospital 64 & -2.2 & 0.01 & $-2.2,-2.2$ & -2.4 & 0.01 & $-2.4,-2.4$ \\
\hline Hospital 03 & -2.2 & 0.02 & $-2.2,-2.2$ & -2.4 & 0.02 & $-2.4,-2.3$ \\
\hline Hospital 14 & -2.2 & 0.02 & $-2.2,-2.1$ & -2.3 & 0.02 & $-2.4,-2.3$ \\
\hline Hospital 63 & -2.1 & 0.01 & $-2.2,-2.1$ & -2.3 & 0.01 & $-2.3,-2.3$ \\
\hline Hospital 59 & -2.1 & 0.02 & $-2.2,-2.1$ & -2.3 & 0.02 & $-2.3,-2.3$ \\
\hline Hospital 48 & -2.1 & 0.01 & $-2.1,-2.1$ & -2.2 & 0.01 & $-2.3,-2.2$ \\
\hline Hospital 46 & -2.0 & 0.02 & $-2.0,-1.9$ & -2.1 & 0.02 & $-2.1,-2.1$ \\
\hline Hospital 56 & -1.9 & 0.01 & $-1.9,-1.9$ & -2.0 & 0.02 & $-2.1,-2.0$ \\
\hline Hospital 37 & -1.8 & 0.01 & $-1.9,-1.8$ & -2.0 & 0.01 & $-2.0,-1.9$ \\
\hline Hospital 52 & -1.5 & 0.01 & $-1.6,-1.5$ & -1.6 & 0.01 & $-1.6,-1.6$ \\
\hline Hospital 32 & -1.5 & 0.00 & $-1.5,-1.5$ & -1.6 & 0.00 & $-1.6,-1.6$ \\
\hline Hospital 39 & -1.4 & 0.01 & $-1.4,-1.4$ & -1.5 & 0.01 & $-1.5,-1.4$ \\
\hline
\end{tabular}

"diversion HHI" tends to 1, the market is likely to be either a monopoly (if the time-elasticity estimated for hospital $j$ is low) or duopoly (if the time-elasticity estimated for hospital $j$ is high). Since the estimated time-elasticities are fairly high while the computed HHI's are rather low, all hospitals in our study sample seem to face strong competition in the market for neurosurgery.

\section{Sensitivity tests}

To test for the robustness of our findings, we use the vector of estimated parameters and corresponding covariance matrix from our conditional logit model to randomly draw 1,000 sets of alternative, equally probable model parameters from a multivariate normal distribution with this vector and matrix as means and covariance, respectively. ${ }^{15}$ For each of these sets of coefficients hospital $j$ 's time-elasticity is computed, resulting in a sample of 1,000 different time-elasticities from which we construct a $95 \%$ confidence interval for the time-elasticity of hospital $j$. Since the estimated time-elasticity is defined as the percent change in number of patients divided by the percent change in travel time, it may depend on the size of the artificial travel time change used. As an additional sensitivity test, using the same sets of alternative model parameters, we therefore also compute hospitals' time-elasticities using a $5 \%$ increase in travel times. The results from both Monte Carlo simulations are summarised in Table 7 and Fig. 1. From the sensitivity tests it follows that the estimated time-elasticities are very robust.

15 This parametric bootstrap is also referred to as the Krinsky-Robb method. The only assumption this method requires is that the estimated coefficients are joint normally distributed (Krinsky and Robb 1986, 1990), which is in fact a statistical property of the conditional logit model. Maximum likelihood estimation of this model under very general conditions provides estimators that are normally distributed. The approximation is reasonably good even in quite small samples (McFadden 1974, pp. 119/120). 


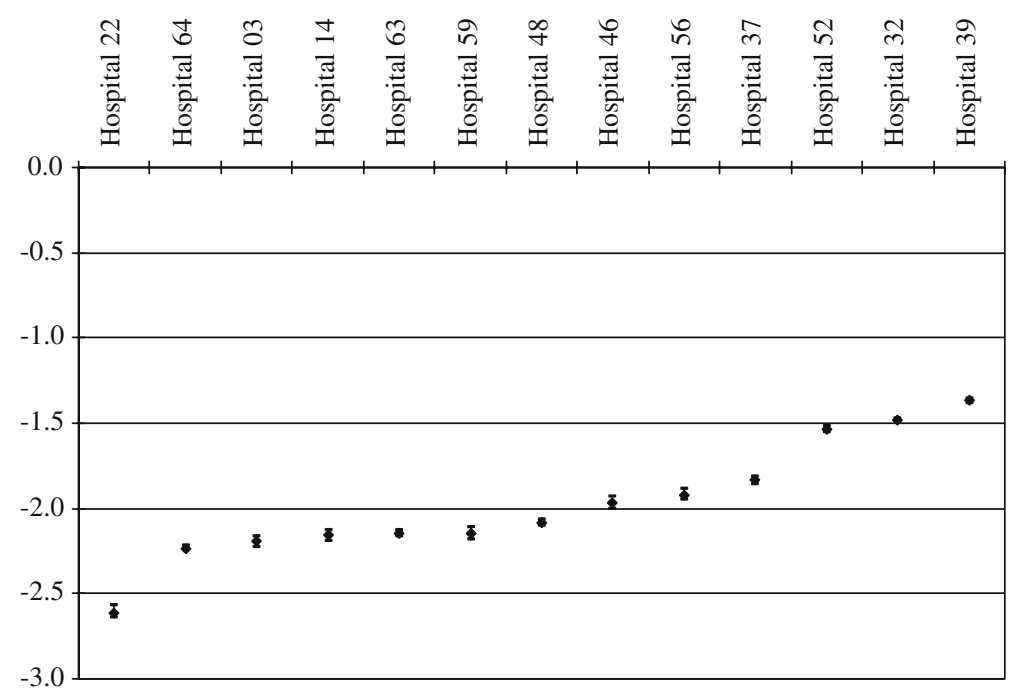

Fig. 1 Estimated time elasticities' robustness (travel time $+10 \%$ )

\section{Concluding remarks}

In several European countries, governments increasingly rely on competition as a strategy to encourage health care providers to reduce prices and increase quality. The success of this strategy depends on consumers' propensity to switch health care providers. Health insurers will not be able to negotiate favourable contracts (i.e. price discounts and/or quality improvements) with health care providers whose demand is rather inelastic.

In this paper we illustrate how to assess the feasibility of competition among hospitals in a setting where patients are insensitive to hospital prices, which is the case in many European countries. When insurance coverage is comprehensive for hospital services and out-of-pocket payments are absent or do not differ across hospitals, travel time functions as the only "price" for patients. In this setting hospitals' relative bargaining power with insurers can be assessed by computing their time-elasticities: the percent change in market share divided by the artificial percent change in travel time.

The time-elasticity approach starts by estimating a conditional logit model of patient hospital choice. Using data from a large Dutch health insurer on non-emergency neurosurgical outpatient hospital visits in 2003, we find that patients are indeed averse to travel. We use the parameter estimates to compute time-elasticities for thirteen Dutch hospitals. We simulate how patients, given their own characteristics, would respond when more travel time is required to visit the hospital, holding all other hospital attributes constant.

From our simulations it follows that the point estimates of hospitals' time-elasticities range from -1.4 to -2.6 . When travel time is raised by $10 \%$ for every patient, the former hospital looses $14 \%$ of its patients to other hospitals while the latter looses as many as $26 \%$ of its patients. Our analysis reveals that overall hospital demand in the Netherlands is rather time-elastic which may suggest that effective hospital competition for neurosurgery is feasible. Estimated time-elasticities differ significantly across individual hospitals. In the market for neurosurgery some hospitals thus face fewer close substitutes than others and therefore have a stronger market position. 
One should keep in mind that this paper analyses hospital substitutability in one particular submarket, defined as an insurer-hospital service pair. The empirical findings are therefore not necessarily representative for hospitals' substitutability in other submarkets. From a conceptual perspective, however, our findings clearly indicate that in a setting where patients' decisions which hospital to visit are based on non-price factors only, the time-elasticity approach can be a useful instrument to assess hospital substitutability that underlies hospital competition. Such information is not only relevant for assessing the feasibility of selective contracting by health insurers, but also for assessing proposed hospital mergers by antitrust enforcement agencies. First, time-elasticities can be used for ruling out hospitals as close substitutes allowing for instance for quick review of mergers not involving close substitutes. Second, by using time-elasticities for ranking hospital substitutes, antitrust analysis can focus on a particular set of hospitals that are the merging hospitals' closest substitutes. The ensuing analysis might then be qualitative and quantitative in nature, but it would be at least focused on the right hospitals.

Acknowledgments The authors thank Paul van der Geest, Wynand van de Ven, Pinar Karaca-Mandic, and two anonymous reviewers for their helpful comments and suggestions. They also gratefully acknowledge Agis for providing the data.

Open Access This article is distributed under the terms of the Creative Commons Attribution Noncommercial License which permits any noncommercial use, distribution, and reproduction in any medium, provided the original author(s) and source are credited.

\section{References}

Bronstein, J. M., \& Morrisey, M. A. (1991). Bypassing rural hospitals for obstetrics care. Journal of Health Politics, Policy and Law, 16(1), 87-118.

Buchmueller, T. (2006). Price and the health plan choices of retirees. Journal of Health Economics, 25(1), 81101.

Burns, L. R., \& Wholey, D. R. (1992). The impact of physician characteristics in conditional choice models for hospital care. Journal of Health Economics, 11(1), 43-62.

Capps, C. S., Dranove, D., Greenstein S., \& Satterthwaite M. (2001), The silent majority fallacy of the Elzinga-Hogarty criteria: A critique and new approach to analyzing hospital mergers, Working Paper 8216, National Bureau of Economic Research (NBER), Cambridge (Mass.).

Capps, C. S., Dranove, D., \& Satterthwaite, M. (2003). Competition and market power in option demand markets. RAND Journal of Economics, 34(4), 737-763.

Elsevier. (2003). De beste ziekenhuizen (The best hospitals), 27 Sept, 38-50.

Folland, S. T. (1983). Predicting hospital market shares. Inquiry, 20(1), 34-44.

Garnick, D. W., Lichtenberg, E., Phibbs, C. S., Luft, H. S., Peltzman, D. J., \& McPhee, S. J. (1989). The sensitivity of conditional choice models for hospital care to estimation technique. Journal of Health Economics, 8(4), 377-397.

Hanson, K., Yip, W. C., \& Hsiao, W. (2004). The impact of quality on the demand for outpatient services in Cyprus. Health Economics, 13(12), 1167-1180.

Hausman, J., \& McFadden, D. (1984). Specification tests for the multinomial logit model. Econometrica, 52(5), 1219-1240.

Hodgkin, D. (1996). Specialized service offerings and patients' choice of hospital: The case of cardiac catheterization. Journal of Health Economics, 15(3), 305-332.

Howard, D. H. (2005). Quality and consumer choice in healthcare: Evidence from kidney transplant. Topics in Economic Analysis \& Policy, 5(1), 1-20.

Krinsky, I., \& Robb, A. L. (1986). On approximating the statistical properties of elasticities. Review of Economics and Statistics, 68(4), 715-719.

Krinsky, I., \& Robb, A. L. (1990). On approximating the statistical properties of elasticities: A correction. Review of Economics and Statistics, 72(1), 189-190.

Lee, H. L., \& Cohen, M. A. (1985). A multinominal logit model for the spatial distribution of hospital utilization. Journal of Business \& Economic Statistics, 3(2), 159-168. 
Martin, S., Rice, N., Jacobs, R., \& Smith, P. (2007). The market for elective surgery: Joint estimation of supply and demand. Journal of Health Economics, 26(2), 263-285.

McFadden, D. (1974). Conditional logit analysis of qualitative choice behavior. In P. Zarembka (Ed.), Frontiers in econometrics (pp. 105-142). New York, NY: Academic Press.

McGuirk, M. A., \& Porell, F. W. (1984). Spatial patterns of hospital utilization: The impact of distance and time. Inquiry, 21(1), 84-95.

Morill, R. L., Earickson, R. J., \& Rees, P. (1970). Factors influencing distances travelled to hospitals. Economic Geography, 46(2), 161-172.

NZa. (2007). Richting geven aan keuzes: Visiedocument (Channelling consumer choice). Utrecht: Dutch Healthcare Authority (NZa).

Phibbs, C. S., Mark, D. H., Luft, H. S., Peltzman-Rennie, D. J., Garnick, D. W., Lichtenberg, E., et al. (1993). Choice of hospital for delivery: A comparison of high-risk and low-risk women. Health Services Research, 28(2), 201-222.

Porell, F. W., \& Adams, E. K. (1995). Hospital choice models: A review and assessment of their utility for policy impact analysis. Medical Care Research and Review, 52(2), 158-195.

Roghmann, K. J., \& Zastowny, T. R. (1979). Proximity as a factor in the selection of health providers: Emergency room visits compared to obstetric admissions and abortions. Social Science \& Medicine, 13((1), 61-63.

Schut, F. T., \& Van de Ven, W. P. M. M. (2005). Rationing and competition in the Dutch health-care system. Health Economics, 14(S1), S59-S74.

Sorensen, A. T. (2003). Insurer-hospital bargaining: Negotiated discounts in post-deregulated Connecticut. Journal of Industrial Economics, 51(4), 469-490.

Tai, W. C., Porell, F. W., \& Adams, E. K. (2004). Hospital choice of rural Medicare beneficiaries: Patient, hospital attributes, and the patient-physician relationship. Health Services Research, 39(6), 1903-1922.

Tay, A. (2003). Assessing competition in hospital care markets: The importance of accounting for quality differentiation. RAND Journal of Economics, 34(4), 786-814.

Town, R. J., \& Vistnes, G. (2001). Hospital competition in HMO networks. Journal of Health Economics, 20(5), 733-753.

Varkevisser, M., Capps, C. S., \& Schut, F. T. (2008). Defining hospital markets for antitrust enforcement: New approaches and their applicability to the Netherlands. Health Economics, Policy and Law, 3(1), 7-29.

Varkevisser, M., \& Van der Geest, S. A. (2007). Why do patients bypass the nearest hospital? An empirical analysis for orthopaedic care and neurosurgery in the Netherlands. European Journal of Health Economics, 8(3), 287-295. 